\title{
Toward Tracing the Tangled Paths of Genes During Development
}

\author{
Evolution, Development, \& the Predictable Genome, by David L. Stern. \\ Greenwood Village: Roberts and Company Publishers, 2011. Pp. xvi+264. H/b \$45.00
}

\author{
Diddahally R. Govindaraju
}

Published online: 6 March 2012

(C) Springer Science+Business Media, LLC 2012

How hereditary material is transformed into a myriad range of phenotypic diversity has preoccupied biologists for at least 150 years. Concerted efforts of many early theoretical and experimental geneticists and evolutionists on the topic led to the modern synthesis in the early 1930s. During this period, however, it was realized that all inherited variation is driven by mutations in the genetic material, and their phenotypic expression is mediated by complex developmental processes. Nonetheless, mechanisms involved in the genotype-developmentphenotype link in relation to environment remained largely speculative for decades after the modern synthesis. Mysteries surrounding developmental phases are gradually being solved, largely due to advances in molecular and developmental biology.

Natural selection operates on individual phenotypes, ultimately affecting genes and their frequencies in populations. During this process, genetic, development, and phenotypic changes may occur simultaneously or in tandem, and might even overlap. Ideally then, it makes sense to consider these tripartite components as complementary aspects of the evolutionary process. Instead, these are generally addressed as largely independent aspects. For instance, mechanisms of gene frequency changes are discussed in population genetics, phenotypic level changes are discussed in evolutionary biology, and the developmental aspects in developmental biology contexts, as if they deal with divergent aspects of biology. Even when discussed, developmental aspects receive a cursory treatment. The present book is a rare exception to this tradition and sets an example showing why the three domains of biology need to be considered simultaneously as equal players in understanding the evolutionary process. The author, David Stern, focuses clearly

D. R. Govindaraju $(\bowtie)$

Beth Israel Deaconess Medical Center, Harvard Medical School, Boston MA, USA

e-mail: drgrajugis@gmail.com on this goal from the very start. For example, the author states that while evolutionary biology attempts to answer the "why" question, developmental biology answers "how" and searches for mechanistic details couched at the gene level. This treatment illuminates the evolutionary process meaningfully.

The book is divided into eight chapters. In the first two introductory chapters, Stern presents the logic for "marrying" evolutionary studies with development. Chapters 3-5 deal with three important, but neglected, genetic concepts: dominance, pleiotropy, and epistasis. In genetics, additive genetic variation is often emphasized, but the phenotypic manifestation of these three factors during development is largely ignored. In particular, Stern's use of enzyme kinetics data to illustrate the role of dominance, the examples of pleiotropy (page 56), and explanations on epistasis are simply elegant, and also represent some of the best I have read on these topics. The next chapter deals with natural selection in relation to populations, the role of population size in fixation of mutations, and population cycles as well as phenotypic plasticity. The role of migration is only briefly mentioned in the notes section. The following chapter, "Pathworks," is in my opinion the best chapter. The author's emphasis on critical nodal points or "hot spots" for evolutionary divergence is central to our understanding of stability in the expression of morphological traits across populations and species. I did not, however, see any reference to Sewall Wright's "path analysis" and its extensions to physiological genetics, population divergence, and speciation. I believe that Wright essentially advocated these views throughout his life. The next chapter, "The predictable genome," which also appears in the book title, is both ambitious and ambiguous. At the outset, it gives the impression that phenotypic effects are predictable from genomic variants. Stern provides some excellent examples to support this point, including Mendel's "wrinkled pea" seed 
and the myostatin gene in Belgian bulls. However, he is cautious about making a general statement supporting many genocentric and deterministic views, as numerous genes have unpredictable phenotypic effects due to pleiotropy and epistasis. Knowledge of the effect size and nature of inheritance (Mendelian or polygenic) of the genomic variants is critical for predictability. Myostatin mutations, for example, have trade-offs and fitness consequences. Finally, the last chapter ends with a historic note on macromutation and mutational hot spots and suggests that accumulation of micromutations plays a more important role in evolution than macromutations. The book has an extensive bibliography spanning evolutionary genetics and developmental genetics. I was a bit disappointed that Waddington's work was not mentioned anywhere in the book. Similarly, excellent examples from human developmental disorders are overlooked.
These small quibbles aside, I applaud the author for his attempt to link evolutionary genetic concepts with modern discoveries in developmental biology. This is a well produced and an insightful book, written in a simple language without cluttering from references and technical details. These are moved to a separate notes section. The author has used simple yet effective illustrations. In view of the recent plethora of genome-wide association studies to understand the genetic architecture of quantitative traits, developmental aspects are often ignored, especially pleiotropy, epistasis, effect sizes of novel mutations and modularity of developmental pathways - consequently misleading many aspects of genetics. This book reminds us all to reevaluate many of our 'gene for' claims in the light of development. In short, this is an excellent and timely work of extraordinary importance for all biologists. 